\title{
Heterogeneity in Microstructural Deterioration Following Spinal Cord Injury
}

Short title: Bone microstructure in spinal cord paralysis

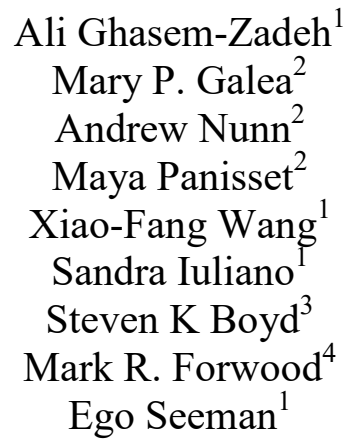

${ }^{1}$ Depts of Medicine and Endocrinology, Austin Health, The University of Melbourne, Melbourne, Australia.

${ }^{2}$ Depts of Medicine and Victorian Spinal Cord Service, Austin Health, The University of Melbourne, Melbourne, Australia.

${ }^{3}$ McCaig Institute for Bone and Joint Health, University of Calgary, Calgary, AB, Canada.

${ }^{4}$ School of Medical Science and Menzies Health Institute Queensland, Griffith University, Gold Coast, Australia.

Correspondence: Ali Ghasem-Zadeh. Dept of Endocrinology, $2^{\text {nd }}$ floor, Centaur Wing, Repatriation Campus, Austin Health, Waterdale Rd, West Heidelberg 3081 Victoria, Australia Tel +6139496 2081, Fax +613 9496 3365, Email: alig@unimelb.edu.au 


\begin{abstract}
Background Modelling and remodelling adapt bone morphology to accommodate strains commonly encountered during loading. If strains exceed a threshold threatening fracture, modelling-based bone formation increases bone volume reducing these strains. If unloading reduces strains below a threshold that inhibits resorption, increased remodellingbased bone resorption reduces bone volume restoring strains, but at the price of compromised bone volume and microstructure. As weight-bearing regions are adapted to greater strains, we hypothesized that microstructural deterioration will be more severe than at regions commonly adapted to low strains following spinal cord injury.
\end{abstract}

Methods We quantified distal tibial, fibula and radius volumetric bone mineral density (vBMD) using high-resolution peripheral quantitative computed tomography in 31 men, mean age 43.5 years (range 23.5-75.0), 12 with tetraplegia and 19 with paraplegia of 0.7 to 18.6 years duration, and 102 healthy age- and sex-matched controls. Differences in morphology relative to controls were expressed as standardized deviation (SD) scores (mean $\pm \mathrm{SD})$. Standardized between-region differences in vBMD were expressed as SDs $(95 \%$ confidence intervals, CI).

Results Relative to controls, men with tetraplegia had deficits in total vBMD of $1.72 \pm 1.38 \mathrm{SD}$ at the distal tibia $(\mathrm{p}<0.001)$ and $-0.68 \pm 0.69 \mathrm{SD}$ at distal fibula $(\mathrm{p}=0.041)$, but not at the distal radius, despite paralysis. Deficits in men with paraplegia were $-2.14 \pm 1.50$ $\mathrm{SD}(\mathrm{p}<0.001)$ at the distal tibia and $-0.83 \pm 0.98 \mathrm{SD}(\mathrm{p}=0.005)$ at the distal fibula while distal radial total vBMD was $0.23 \pm 1.02(\mathrm{p}=0.371)$, not significantly increased, despite upper limb mobility. Comparing regions, in men with tetraplegia, distal tibial total vBMD was $1.04 \mathrm{SD}$ $(95 \%$ CI $0.07,2.01)$ lower than at the distal fibula $(p=0.037)$ and 1.51 SD $(95 \%$ CI 0.45 , 2.57) lower than at the distal radius $(\mathrm{p}=0.007)$; the latter two sites did not differ from each other. Results were similar in men with paraplegia, but total vBMD at the distal fibula was $1.06 \mathrm{SD}(95 \%$ CI $0.35,1.77)$ lower than at the distal radius $(\mathrm{p}=0.004)$.

Conclusion Microarchitectural deterioration following spinal cord injury is heterogeneous, perhaps partly because strain thresholds regulating the cellular activity of mechano-transduction are region specific.

Keywords Cortical Bone, HR-pQCT, Microstructure, Paralysis, Spinal Cord Injury, Trabecular bone, Unloading, Weight-bearing, 


\section{Introduction}

Bone has paradoxical properties of strength for loading yet lightness for mobility, stiffness for leverage yet flexibility for energy absorption. ${ }^{(1)}$ These properties are achieved by bone modelling and remodelling, a cellular machinery that adapts the spatial distribution of bone's mineralized matrix volume to its usual loading circumstances. ${ }^{(2)}$ For example, in an individual, adjacent cross sections of a long bone are assembled using similar volumes of mineralized matrix. ${ }^{(3)}$ Differences in the external size, shape and internal microarchitecture of each adjacent cross section along the bone are the result of differences in the volumes of the medullary and intracortical canals, not the volume of mineralized matrix. ${ }^{(4)}$

Mass is minimized in larger cross sections by assembling them with relatively more void volume, not more matrix volume. The constant bone matrix volume is distributed radially by excavating a larger medullary canal producing a thinner cortex. The thinner cortex is distributed around a larger perimeter preserving cortical area and so compressive strength. The greater radial distribution of cortical mass increases bending and torsional strength disproportionately to the increase in matrix volume. ${ }^{(5)}$ Smaller cross sections achieve robusticity by being assembled with a relatively thicker cortex and relatively less void volume, not less matrix volume. ${ }^{(6)}$

The diversity in external bone size, shape, and architecture is largely the result of genetic variance. ${ }^{(7)}$ However, focal differences in loading also contribute to this structural diversity because bone is mechanosensitive. ${ }^{(2)}$ Bone adapts its structural design to accommodate a range of commonly encountered loading circumstances. These structural adaptations ensure that bone strains during loading remain within a range that does not threaten fracture during usual activities. ${ }^{(2)}$ These strain ranges and the thresholds may also be region specific and selected for by genetic factors. ${ }^{(8)}$ If strains exceed a threshold associated with a high risk of fracture, modelling and remodelling restore strains by increasing or redistributing total bone volume. ${ }^{(2,9)}$ When unloading occurs, strains fall below a threshold inhibiting resorption, resorptive modelling or negatively balanced remodelling restore strains by reducing bone volume and compromising bone microstructural strength. ${ }^{(2,10)}$

Bone loss following spinal cord injury occurs below the level of the injury but the severity of bone loss and the cellular activity adapting bone to its loading are region specific. ${ }^{(11-15)}$ Weight bearing regions like the tibia are subjected to higher loads and strain rates than less weight bearing regions, like the fibula, ${ }^{(16)}$ or non-weight bearing regions like the radius. Bone loss reduces bone volume and disrupts the microarchitecture of the remaining bone volume. The microarchitectural deterioration reduces bone strength as a power function of the bone loss causing it. ${ }^{(17-19)}$ Bone mineral density (BMD) measurements do not capture microstructural deterioration and so, bone fragility may be present in patients with spinal cord injury with osteopenia or so-called normal BMD. We therefore hypothesized that weight bearing regions will have lost more bone and structural integrity than less weightbearing or non-weight bearing bones after spinal cord injury.

\section{Methods}

Participants We recruited 12 men with tetraplegia mean age 44.6 years (range 25.9 - 67.2) and 19 men with paraplegia, mean age 42.9 years (range 23.5 - 75.0) with injury of various grades. $^{(20)}$ Men with tetraplegia were studied 6.5 years, range $0.7-15.6$, and men with paraplegia were studied 6.5 years (range 1.6 - 18.6) after the injury and compared with 102 age matched healthy male controls. Men aged under 18 years, with a history of distal tibia and radius fractures or treatment for osteoporosis were excluded. Participants provided 
informed consent prior to participation. The study was approved by the Austin Health Research Ethics Committee.

Image acquisition and analysis Image acquisition, reconstruction, analysis and validation have been reported. ${ }^{(21,22)}$ Participants had distal radius, distal tibia and distal fibula microstructure quantified using HR-pQCT (Scanco Medical, Switzerland) at Austin Health. A region of interest of $9.02 \mathrm{~mm}$ was acquired starting $9.5 \mathrm{~mm}$ or $22.5 \mathrm{~mm}$ proximal to the joint line of the distal radius and tibia, respectively. The 49 most proximal slices of a region of interest were chosen for analysis. Images were reconstructed at a resolution of $82 \mu \mathrm{m}$. Total, cortical and medullary areas, and volumetric bone mineral density (vBMD) of total, trabecular and cortical compartments, trabecular number, thickness, separation, cortical porosity, and matrix mineral density were quantified using StrAx1.0 algorithm (Strax Corp, Melbourne, Australia).

A non-threshold based method selects attenuation profile curves and segments (separates) bone from background and cortical from trabecular bone. ${ }^{(23)}$ Profile curves are produced as photons are attenuated by mineralized bone. Bone edges are identified as the beginning and end of the rise and fall of the attenuation profie. Analyzing $\sim 3600$ profiles around the perimeter of each cross-sectional slice segments the compartments. Porosity is quantified as the average void volume fraction of all voxels within each compartment.

Agreement between HR-pQCT and the gold standard ( $\mu \mathrm{CT}$ of cadaveric specimens imaged at $19 \mu \mathrm{m}$ voxel size) ranged from $\mathrm{R}^{2}=0.87$ to 0.99 . Precision error in vivo was $0.54 \%$ for porosity. Quality control for the performance of HR-pQCT was based on daily scanning phantoms containing hydroxyapatite (densities $0,100,200,400$, and $800 \mathrm{mgHA} / \mathrm{cm}^{3}$ ) (QRM, Germany). Radiation exposure is 3-5 $\mu \mathrm{Sv}$ per measurement. ${ }^{(4,22)}$ The coefficients of variation $(\mathrm{CV})$ of the measurements ranged from $0.54 \%$ to $3.98 \%{ }^{(23)}$ Inhomogeneity of Trabecular Network is calculated based on the standard deviation of the spacing between mid-axis (ridges) of the trabeculae. Marching cubes and ruler intercept methods were applied to quantify trabecular Structural Model Index (SMI), Connectivity Density (ConnD), and Bone Surface (BS) using specified image analysis algorithm, developed by the manufacturer. $^{(24,25)}$

Bone strength Micro finite element analysis ( $\mu \mathrm{FEA}$ ) was used to estimate Failure Load (FL) using the FAIM software (version 8.0). A uniaxial loading with a tissue elastic modulus of $6,829 \mathrm{MPa}$, a Poisson's ratio of 0.3 , a critical strain of 7000 micro-strain and a critical volume of $2 \%$, were defined. A $1 \%$ apparent strain was applied, and failure load is calculated as the load when $2 \%$ of elements exceeded the critical strain. ${ }^{(25,26)}$

Statistical analysis Statistical analyses were performed using STATA version 14.2 (StataCorp. 2018. Stata Statistical Software. College Station, TX: StataCorp LP). Normality was checked using the Shapiro-Wilk normality test. The Welch's t-test were used to compare traits between cases and controls. Two-tailed p-values $<0.05$ denoted statistical significance. Results were expressed as mean and standard deviation. Differences in standardized terms among sites were expressed as a SD adjusted for age based on simple linear regression.

\section{Results}

\section{Weight bearing regions: distal tibia and distal fibula}

Men with tetraplegia and men with paraplegia had reduced total, cortical and trabecular vBMD at the distal tibia relative to healthy controls (Table 1). Cortical porosity was higher, 
and cortical thickness and matrix mineral density were lower, relative to healthy controls. Trabecular vBMD was reduced due to fewer, thinner, more rod-like and more separated trabeculae, with loss of trabecular surface area and connectivity, and greater inhomogeneity of the trabecular network (Figure 1). Failure load was reduced. Distal tibial vBMD was lower and cortical porosity was higher at later than earlier follow-up times (Figure 2). At the distal fibula, total VBMD was lower in men with tetraplegia and men with paraplegia relative to controls, but with deficits confined to trabecular vBMD produced by fewer, more separated trabeculae, with loss of homogeneity in distribution (Table 2).

\section{Non-weight bearing region: distal radius}

Despite the presence of tetraplegia, no deficits in distal radial total, cortical or trabecular vBMD were present. Trabecular thickness was increased in men with tetraplegia and men with paraplegia, and matrix mineral density was reduced, relative to healthy controlsTrabecular number and homogeneity were not deteriorated. Nor did failure load differ from controls (Table 3).

\section{Comparisons of regions, cortical and trabecular bone}

Table 4 shows the standardized mean difference between region with their $95 \%$ confidence intervals. Figure 3 illustrates these differences with their $p$ values. Deficits in total, cortical and trabecular vBMD were greater at the distal tibia than distal fibula or distal radius. Deficits were statistically significant in several, but not all, comparisons. Deficits were also significantly greater in some, but not all, comparisons of the distal fibula versus distal radius.

\section{Discussion}

The purpose of this study was to quantify the microstructural deterioration of the distal metaphyseal regions of the appendicular skeleton in individuals with spinal cord injury. Relative to controls, men with tetraplegia or paraplegia had increased cortical porosity, reduced cortical thickness and matrix mineral density, complete loss of trabecular plates, reduced trabecular surface area, connectivity and loss of homogeneity of the interconnected plates seen as patching fallout of trabeculae. These changes resulted in loss of estimated strength at the distal tibia, a weight bearing site. More modest deficits were found at the distal fibula, a weight bearing site subjected to lower stresses during loading. ${ }^{(27)}$

Most published studies, like those cited in a meta-analysis, ${ }^{(12)}$ focussed on the reduction in BMD, a measurement that does not capture the heterogeneity in cortical and trabecular morphology within and between regions of the skeleton. We report a hierarchy of deficits. The tibia experienced the greatest loss, followed by the fibula, with the least loss from the radius. These observations are consistent with the relative reduction in loading following spinal cord injury, ${ }^{(12)}$ with the tibia experiencing the greatest unloading, and the radius the least unloading relative to their customary loading.

We observed deficits in cortical and trabecular vBMD at the distal tibia, but deficits in vBMD at the distal fibula were confined to trabecular bone, and were less than the deficits at the distal tibia. The fibula is slender and largely cortical. Cortical bone has a lower surface area/matrix volume ratio than trabecular bone and so is less susceptible to being remodelled than trabecular bone following unloading. ${ }^{(12)}$ As the fibula is subjected to lower strains than 
the tibia, we suggest that paralysis may have resulted in strains deviating less from their customary strain environment than the deviation of strains usually encountered at the tibia. ${ }^{(16)}$ Consequently, loss of trabecular bone may have been sufficient to restore strains without the need to compromise cortical bone.

Eser et al reported deficits in vBMD at the distal femur and proximal tibia were confined to trabecular bone despite these weight-bearing bones being subjected to higher strains than the radius.$^{(17)}$ The larger trabecular surface area/matrix volume ratio at these locations may also make these regions susceptible to more rapid trabecular than cortical bone loss.

Finding uncompromised distal radial microstructure in men with tetraplegia was unanticipated. However, scrutiny of the literature supports the likely validity of this observation. For example, in a meta-analysis, ${ }^{(12)}$ lower limb bones had greater deficits in bone mass than upper extremity bones. Eser et al report rapid decreases in vBMD at the distal femur and tibia in patients with tetraplegia or paraplegia with little loss of bone at the distal radius in patients with paraplegia (as expected) and in the smaller number of patients with tetraplegia. ${ }^{(28)}$ Studies of astronauts demonstrate preservation of radial bone, a structure commonly subjected to lower loads than weight bearing structures. ${ }^{(29)}$ Modlesky et al reported reduced distal femur and proximal tibia trabecular density using high-resolution magnetic resonance imaging in 10 men with long-term complete spinal cord injury compared to 8 controls. Despite complete SCI injury between C6-L1, radius BMD was not reduced relative to controls, as confirmed in this, and other studies. ${ }^{(30)}$

No deficits at the distal radius of men with paraplegia were expected but evidence of higher vBMD in men with paraplegia than in healthy controls or men with tetraplegia might be expected due to mechanical usage for propulsion of a wheelchair. This was not observed. However, Goktepe et al reported higher distal radial BMD in elite athletes with paraplegia, not sedentary subjects with paraplegia, an observation suggesting greater than usually encountered strains are needed to stimulate bone formation in this non-weight bearing bone. $^{(31)}$ The higher distal radial trabecular thickness in both tetraplegia and paraplegia than controls is likely to be due to preferential loss of thinner trabeculae as reported following rapid perimenopausal bone loss. ${ }^{(32)}$

Likewise, in a study of dogs subjected to 40 weeks of immobilization, the greatest loss of bone occurred at the metacarpals (50\%), compared to humeri which lost the least bone $(28 \%) .{ }^{(10)}$ Although the forelimbs of quadrupeds are weight-bearing, the distal limb bones experience greater peak stresses during locomotion than the proximal humerus. ${ }^{(33)}$ Hsieh and colleagues reported using an in vivo loading of the ulna in rats that threshold strains are largest distally, where locomotor strains are typically higher than proximally. There was a correlation between greater peak strains and greater periosteal bone formation once a threshold was exceeded. ${ }^{(34)}$ Schulte et al also reported that bone formation in mice occurs at sites of high local mechanical strain while bone resorption occurred at sites of low local strain. ${ }^{(35)}$ Christen et al demonstrate a linear relationship between focal remodelling and local loading determined by micro finite element analysis in humans but non-mechanically determined component of this cellular activity fulfilling other demands for remodelling. ${ }^{(36)}$

We propose that the heterogeneity of the morphological changes following spinal cord injury is likely to reflect the nature of bone as a mechanosensitive organ. Harold Frost, and many investigators since, suggested that bone modelling and remodelling adapt bone morphology to differences in microstrain imposed by unloading, physiological loading, 
overloading and pathological loading. ${ }^{(2,5,8,9,15,37)}$ The mechanisms responsible for mechanotransduction are now better understood (Figure 4). During loading, bone matrix deformation (strain) generates lacuna-canalicular fluid flow which induces shear stress on osteocytes, down regulating sclerostin, an inhibitor of bone formation and stimulator of bone resorption. ${ }^{(38,39)}$ When sclerostin expression is suppressed, bone formation increases focally. ${ }^{(39)}$ Concurrently, resorptive modelling, or negatively balanced remodelling, occur at other locations. This alters the spatial distribution of the mineralized matrix, adapting a region's microarchitecture to the new loading circumstances while avoiding an increase in total bone volume, which is energy requiring. ${ }^{(1,2,40)}$

Reduced loading in individuals with spinal cord injury reduces canalicular fluid flow. Reduced strains upregulate sclerostin leading to reduced bone formation, increased bone resorption, bone loss and a reduction in bone volume which restores local strains at the price of compromised bone volume, microarchitecture and bone strength. ${ }^{(41)}$ Sclerostin levels were not available in the present study. Published studies suggest that changes in sclerostin levels depend on the type and duration of SCI. ${ }^{(42,43)}$ For example, Battaglino et al reported that sclerostin levels were greatest in subjects with short-term SCI ( $\leq 5$ years) and decreased during the first 5 years post-injury. ${ }^{(42)}$ By contrast, Maimoun et al report lower concentration of sclerostin, ${ }^{(43)}$ a finding that should be associated with increased bone formation. Circulating concentrations may not be appropriate surrogates for the local action of this osteocyte product as reduced levels should be associated with increased bone formation.

In addition to changes in bone volume and microstructure, matrix mineral density was lower in men with tetraplegia and paraplegia, despite the absence of changes in morphology at the distal radius. This observation suggests that rapid remodelling has a systemic effect compromising bone properties above the level of the spinal cord injury. Shen et al reported decreased mineral to collagen matrix ratio in the humeri of paraplegic mice. ${ }^{(44)}$ This rapid remodelling may underly the so-called regional acceleratory phenomenon reported by Frost. $^{(45)}$

Stiffness, or resistance to bending, is a function of the degree of matrix mineralization and bone geometry, with the cross-sectional moment of inertia capturing the distribution of mass around the axis of loading. ${ }^{(5)}$ As stiffness is determined by material and structural properties, both can vary independently, as matrix mineralization and microstructure did here. The change was greater at the distal tibia where both microstructure and matrix mineral density were compromised, contributing to the greater decline in estimated failure load.

Prevention of musculoskeletal deterioration following spinal cord injury remains challenging and has been attempted using functional electrical stimulation (FES)-rowing. Gibbons et al. report a case study of a patient with SCI in whom they suggest distal tibial bone loss measured using HR-pQCT was attenuated. ${ }^{(46)}$ Draghici et al. measured distal tibia and distal radial microstructure also using HR- pQCT in an uncontrolled cross-sectional study of 13 men with SCI. ${ }^{(47)}$ Time since injury, total distance rowed, and peak foot force together predicted trabecular, not cortical, morphology. These studies are difficult to interpret due to issues in design and execution. However, in a 6-month controlled prospective study of FES-cycling 30-min 3 times weekly initiated 4.5 weeks post SCI in 19 para- and tetraplegic patients compared with 19 patients with SCI left untreated, no evidence of attenuation of bone loss was observed. ${ }^{(48)}$ By contrast, Fang et al reported that 20 individuals with SCI treated for 12 months with FES-assisted rowing plus zoledronate 
improved distal femur stiffness by $4.6 \%$ estimated using finite element analysis, while stiffness decreased by $13.9 \%$ in those treated with rowing alone. No benefits were observed in either group at the proximal tibia. No group received drug therapy alone or no intervention. ${ }^{(49)}$ In an uncontrolled study, Frotzler et al. reported improvement in thigh cross sectional area, distal femoral epiphysis trabecular and total BMD following 12 months of 3.7 hourly sessions per week FES-cycling in 11 subjects with complete SCI 11.0 years post injury. ${ }^{(50)}$ Bone parameters in the femoral shaft decreased. Further studies are needed to address this therapeutic challenge.

This study has several limitations. This was a cross-sectional data with limited number of individuals with spinal cord injury and absence of data in the early months following spinal cord injury. For these reasons we could not document the precise temporal patterns of bone loss in each region. However, Edwards et al reported early rapid bone loss of 3-5\% per month. ${ }^{(51)}$ Similarly, Eser et al reported trabecular and cortical bone loss for the distal epiphyses and midshafts of the femur, tibia, and radius measured using peripheral quantitative computed tomography in 24 tetraplegic and 65 paraplegic men with SCI of 2 months and 50 years duration. ${ }^{(17)}$ Bone mass and cortical area of the diaphyses, showed an exponential decrease with time since injury reaching a plateau after 3-8 years. While the upper limbs are not loaded by body weight, about $50 \%$ of this small group of subjects had level D grade ASIA impairment scale, in which most of the muscles are spared below the level of injury, an effect that may partly account for the preservation of bone structure. Analysis of cortical porosity of the fibula was not available. Height and weight measurements were not available so matching with controls was confined to age and sex. While unloading due to spinal cord injury plays a most important role in bone loss, bone loss is multifactorial. ${ }^{(12,52)}$ Coexisting autonomic dysfunction was not quantified in this study and may contribute to bone loss, but few, if any, studies have quantified the contribution of the hemodynamic changes that may influence the skeleton. ${ }^{(53,54)}$

In summary, fractures are common in patients with spinal cord injury and often occur at metaphyseal regions. ${ }^{(55)}$ Bone fragility is the result of bone loss that is region specific and is, in part, a function of the change in mechanical load imposed on the region. Following unloading caused by spinal cord injury, weight bearing regions, adapted to accommodate greater peak strains and strain rates, are at higher risk for bone loss and microarchitectural deterioration during unloading than non-weight bearing regions that are adapted to lower peak strains and strain rates. These observations highlight the site specificity of the strain thresholds regulating the cellular activity of mechano-transduction. The rapidity of the change and irreversible microarchitectural deterioration suggest prompt intervention with antiresorptive, anabolic therapy, or both, warrants consideration. 


\section{Disclosures}

A Ghasem-Zadeh is renumerated as image analyst and shareholder in StraxCorp. E Seeman has received research support and lectured at national and international meetings funded by from Allergan, Amgen, Asahi pharmaceuticals, Eli Lilly, and MSD. He is a director of the board, renumerated as Chief Medical Officer, shareholder in StraxCorp, and is one of the inventors of the StrAx1.0 algorithm. S Boyd is a co-founder and co-owner of Numerics88 Solutions Inc, creator of finite element.

\section{Authors' roles}

Study design and conduct: AGZ, ES, MPG and AN. Data collection: AGZ, XFW, SI, MP. Responsibility for StrAx analysis: AGZ, Responsibility for finite element analysis: SB. Statistical analyses: AGZ, and XFW. Drafting manuscript: AGZ, MPG and ES. Data interpretation and approving final version of manuscript: AGZ, MPG, AN, MP, XFW, SI, $\mathrm{SB}, \mathrm{MRF}$ and ES.

\section{References}

1. Currey JD. The mechanical adaptations of bones. Princeton, N.J.: Princeton University Press; 1984. viii, 294 p. p.

2. Frost HM. Bone "mass" and the "mechanostat": a proposal. Anat Rec. Sep 1987;219(1):19. Epub 1987/09/01.

3. Zebaze RM, Jones A, Welsh F, Knackstedt M, Seeman E. Femoral neck shape and the spatial distribution of its mineral mass varies with its size: Clinical and biomechanical implications. Bone. Aug 2005;37(2):243-52. Epub 2005/06/09.

4. Ghasem-Zadeh A, Burghardt A, Wang XF, Iuliano S, Bonaretti S, Bui M, et al. Quantifying sex, race, and age specific differences in bone microstructure requires measurement of anatomically equivalent regions. Bone. Aug 2017;101:206-13. Epub 2017/05/16.

5. Forwood MR. Mechanical effects on the skeleton: are there clinical implications? Osteoporos Int. 2001;12(1):77-83. Epub 2001/04/18.

6. Ruff CB, Trinkaus E, Walker A, Larsen CS. Postcranial robusticity in Homo. I: Temporal trends and mechanical interpretation. Am J Phys Anthropol. May 1993;91(1):21-53. Epub 1993/05/01.

7. Bjornerem A, Bui M, Wang X, Ghasem-Zadeh A, Hopper JL, Zebaze R, et al. Genetic and environmental variances of bone microarchitecture and bone remodeling markers: a twin study. J Bone Miner Res. Mar 2015;30(3):519-27. Epub 2014/11/20.

8. Robling $\mathrm{AG}$, Turner $\mathrm{CH}$. Mechanotransduction in bone: genetic effects on mechanosensitivity in mice. Bone. Nov 2002;31(5):562-9. Epub 2002/12/13.

9. Mosley JR, March BM, Lynch J, Lanyon LE. Strain magnitude related changes in whole bone architecture in growing rats. Bone. Mar 1997;20(3):191-8. Epub 1997/03/01.

10. Uhthoff HK, Jaworski ZF. Bone loss in response to long-term immobilisation. J Bone Joint Surg Br. Aug 1978;60-B(3):420-9. Epub 1978/08/01.

11. Roberts D, Lee W, Cuneo RC, Wittmann J, Ward G, Flatman R, et al. Longitudinal study of bone turnover after acute spinal cord injury. J Clin Endocrinol Metab. Feb 1998;83(2):415-22. Epub 1998/02/19.

12. Jiang SD, Jiang LS, Dai LY. Mechanisms of osteoporosis in spinal cord injury. Clin Endocrinol (Oxf). Nov 2006;65(5):555-65. Epub 2006/10/24. 
13. Maimoun L, Fattal C, Micallef JP, Peruchon E, Rabischong P. Bone loss in spinal cordinjured patients: from physiopathology to therapy. Spinal Cord. Apr 2006;44(4):203-10. Epub 2005/09/15.

14. Biering-Sorensen F, Bohr HH, Schaadt OP. Longitudinal study of bone mineral content in the lumbar spine, the forearm and the lower extremities after spinal cord injury. European journal of clinical investigation. Jun 1990;20(3):330-5. Epub 1990/06/01.

15. Kuruvilla SJ, Fox SD, Cullen DM, Akhter MP. Site specific bone adaptation response to mechanical loading. J Musculoskelet Neuronal Interact. Jan-Mar 2008;8(1):71-8. Epub 2008/04/10.

16. Wang Q, Whittle M, Cunningham J, Kenwright J. Fibula and its ligaments in load transmission and ankle joint stability. Clin Orthop Relat Res. Sep 1996(330):261-70. Epub 1996/09/01.

17. Eser P, Frotzler A, Zehnder Y, Wick L, Knecht H, Denoth J, et al. Relationship between the duration of paralysis and bone structure: a pQCT study of spinal cord injured individuals. Bone. May 2004;34(5):869-80. Epub 2004/05/04.

18. Schaffler MB, Burr DB. Stiffness of compact bone: effects of porosity and density. J Biomech. 1988;21(1):13-6. Epub 1988/01/01.

19. Coupaud S, Gislason MK, Purcell M, Sasagawa K, Tanner KE. Patient-specific bone mineral density distribution in the tibia of individuals with chronic spinal cord injury, derived from multi-slice peripheral Quantitative Computed Tomography (pQCT) - A cross-sectional study. Bone. Apr 2017;97:29-37. Epub 2016/12/31.

20. Maynard FM, Jr., Bracken MB, Creasey G, Ditunno JF, Jr., Donovan WH, Ducker TB, et al. International Standards for Neurological and Functional Classification of Spinal Cord Injury. American Spinal Injury Association. Spinal Cord. May 1997;35(5):266-74. Epub 1997/05/01.

21. Wang XF, Wang Q, Ghasem-Zadeh A, Evans A, McLeod C, Iuliano-Burns S, et al. Differences in macro- and microarchitecture of the appendicular skeleton in young Chinese and white women. J Bone Miner Res. Dec 2009;24(12):1946-52. Epub 2009/05/23.

22. Geusens P, Chapurlat R, Schett G, Ghasem-Zadeh A, Seeman E, de Jong J, et al. Highresolution in vivo imaging of bone and joints: a window to microarchitecture. Nat Rev Rheumatol. May 2014;10(5):304-13. Epub 2014/03/07.

23. Zebaze R, Ghasem-Zadeh A, Mbala A, Seeman E. A new method of segmentation of compact-appearing, transitional and trabecular compartments and quantification of cortical porosity from high resolution peripheral quantitative computed tomographic images. Bone. May 2013;54(1):8-20. Epub 2013/01/22.

24. Hildebrand T, Laib A, Muller R, Dequeker J, Ruegsegger P. Direct three-dimensional morphometric analysis of human cancellous bone: microstructural data from spine, femur, iliac crest, and calcaneus. J Bone Miner Res. Jul 1999;14(7):1167-74. Epub 1999/07/15.

25. Whittier DE, Boyd SK, Burghardt AJ, Paccou J, Ghasem-Zadeh A, Chapurlat R, et al. Guidelines for the assessment of bone density and microarchitecture in vivo using highresolution peripheral quantitative computed tomography. Osteoporos Int. May 262020. Epub 2020/05/28.

26. Samelson EJ, Broe KE, Xu H, Yang L, Boyd S, Biver E, et al. Cortical and trabecular bone microarchitecture as an independent predictor of incident fracture risk in older women and men in the Bone Microarchitecture International Consortium (BoMIC): a prospective study. Lancet Diabetes Endocrinol. Jan 2019;7(1):34-43. Epub 2018/12/07.

27. Funk JR, Rudd RW, Kerrigan JR, Crandall JR. The effect of tibial curvature and fibular loading on the tibia index. Traffic Inj Prev. Jun 2004;5(2):164-72. Epub 2004/06/19.

28. Eser P, Frotzler A, Zehnder Y, Schiessl H, Denoth J. Assessment of anthropometric, systemic, and lifestyle factors influencing bone status in the legs of spinal cord injured individuals. Osteoporos Int. Jan 2005;16(1):26-34. Epub 2004/05/13. 
29. Vico L, van Rietbergen B, Vilayphiou N, Linossier MT, Locrelle H, Normand M, et al. Cortical and Trabecular Bone Microstructure Did Not Recover at Weight-Bearing Skeletal Sites and Progressively Deteriorated at Non-Weight-Bearing Sites During the Year Following International Space Station Missions. J Bone Miner Res. Oct 2017;32(10):2010-21. Epub 2017/06/03.

30. Modlesky CM, Majumdar S, Narasimhan A, Dudley GA. Trabecular bone microarchitecture is deteriorated in men with spinal cord injury. J Bone Miner Res. Jan 2004;19(1):48-55. Epub 2004/02/03.

31. Goktepe AS, Yilmaz B, Alaca R, Yazicioglu K, Mohur H, Gunduz S. Bone density loss after spinal cord injury: elite paraplegic basketball players vs. paraplegic sedentary persons. American journal of physical medicine \& rehabilitation. Apr 2004;83(4):279-83. Epub 2004/03/17.

32. Bjornerem A, Wang X, Bui M, Ghasem-Zadeh A, Hopper JL, Zebaze R, et al. MenopauseRelated Appendicular Bone Loss is Mainly Cortical and Results in Increased Cortical Porosity. J Bone Miner Res. Apr 2018;33(4):598-605. Epub 2017/12/09.

33. Biewener AA. Bone strength in small mammals and bipedal birds: do safety factors change with body size? J Exp Biol. Jun 1982;98:289-301. Epub 1982/06/01.

34. Hsieh YF, Robling AG, Ambrosius WT, Burr DB, Turner CH. Mechanical loading of diaphyseal bone in vivo: the strain threshold for an osteogenic response varies with location. J Bone Miner Res. Dec 2001;16(12):2291-7. Epub 2002/01/05.

35. Schulte FA, Ruffoni D, Lambers FM, Christen D, Webster DJ, Kuhn G, et al. Local mechanical stimuli regulate bone formation and resorption in mice at the tissue level. PLoS One. 2013;8(4):e62172. Epub 2013/05/03.

36. Christen P, Ito K, Ellouz R, Boutroy S, Sornay-Rendu E, Chapurlat RD, et al. Bone remodelling in humans is load-driven but not lazy. Nat Commun. Sep 11 2014;5:4855. Epub 2014/09/12.

37. McNamara LM, Prendergast PJ. Bone remodelling algorithms incorporating both strain and microdamage stimuli. J Biomech. 2007;40(6):1381-91. Epub 2006/08/26.

38. Turner $\mathrm{CH}$, Forwood MR, Otter MW. Mechanotransduction in bone: do bone cells act as sensors of fluid flow? FASEB J. Aug 1994;8(11):875-8. Epub 1994/08/01.

39. Robling AG, Niziolek PJ, Baldridge LA, Condon KW, Allen MR, Alam I, et al. Mechanical stimulation of bone in vivo reduces osteocyte expression of Sost/sclerostin. J Biol Chem. Feb 29 2008;283(9):5866-75. Epub 2007/12/20.

40. Bass SL, Saxon L, Daly RM, Turner CH, Robling AG, Seeman E, et al. The effect of mechanical loading on the size and shape of bone in pre-, peri-, and postpubertal girls: a study in tennis players. J Bone Miner Res. Dec 2002;17(12):2274-80. Epub 2002/12/10.

41. Tian X, Jee WS, Li X, Paszty C, Ke HZ. Sclerostin antibody increases bone mass by stimulating bone formation and inhibiting bone resorption in a hindlimb-immobilization rat model. Bone. Feb 2011;48(2):197-201. Epub 2010/09/21.

42. Battaglino RA, Sudhakar S, Lazzari AA, Garshick E, Zafonte R, Morse LR. Circulating sclerostin is elevated in short-term and reduced in long-term SCI. Bone. Sep 2012;51(3):600-5. Epub 2012/05/12.

43. Maimoun L, Ben Bouallegue F, Gelis A, Aouinti S, Mura T, Philibert P, et al. Periostin and sclerostin levels in individuals with spinal cord injury and their relationship with bone mass, bone turnover, fracture and osteoporosis status. Bone. Oct 2019;127:612-9. Epub 2019/07/28.

44. Shen J, Fan L, Yang J, Shen AG, Hu JM. A longitudinal Raman microspectroscopic study of osteoporosis induced by spinal cord injury. Osteoporos Int. Jan 2010;21(1):81-7. Epub 2009/05/14.

45. Frost HM. The regional acceleratory phenomenon: a review. Henry Ford Hosp Med J. 1983;31(1):3-9. Epub 1983/01/01.

46. Gibbons RS, Beaupre GS, Kazakia GJ. FES-rowing attenuates bone loss following spinal cord injury as assessed by HR-pQCT. Spinal cord series and cases. 2016;2:15041. Epub 2017/01/06. 
47. Draghici AE, Taylor JA, Bouxsein ML, Shefelbine SJ. Effects of FES-Rowing Exercise on the Time-Dependent Changes in Bone Microarchitecture After Spinal Cord Injury: A CrossSectional Investigation. JBMR Plus. Sep 2019;3(9):e10200. Epub 2019/11/02.

48. Eser P, de Bruin ED, Telley I, Lechner HE, Knecht H, Stüssi E. Effect of electrical stimulation-induced cycling on bone mineral density in spinal cord-injured patients. European journal of clinical investigation. May 2003;33(5):412-9. Epub 2003/04/26.

49. Fang Y, Morse LR, Nguyen N, Battaglino RA, Goldstein RF, Troy KL. Functional electrical stimulation (FES)-assisted rowing combined with zoledronic acid, but not alone, preserves distal femur strength and stiffness in people with chronic spinal cord injury. Osteoporos Int. Sep 4 2020. Epub 2020/09/06.

50. Frotzler A, Coupaud S, Perret C, Kakebeeke TH, Hunt KJ, Donaldson NN, et al. Highvolume FES-cycling partially reverses bone loss in people with chronic spinal cord injury. Bone. Jul 2008;43(1):169-76. Epub 2008/04/29.

51. Edwards WB, Schnitzer TJ, Troy KL. Bone mineral and stiffness loss at the distal femur and proximal tibia in acute spinal cord injury. Osteoporos Int. Mar 2014;25(3):1005-15. Epub 2013/11/06.

52. Jiang SD, Dai LY, Jiang LS. Osteoporosis after spinal cord injury. Osteoporos Int. Feb 2006;17(2):180-92. Epub 2005/10/12.

53. Chantraine A, van Ouwenaller C, Hachen HJ, Schinas P. Intra-medullary pressure and intra-osseous phlebography in paraplegia. Paraplegia. Nov 1979;17(4):391-9. Epub 1979/11/01.

54. Shim SS, Hawk HE, Yu WY. The relationship between blood flow and marrow cavity pressure of bone. Surgery, gynecology \& obstetrics. Sep 1972;135(3):353-60. Epub 1972/09/01.

55. Zehnder Y, Luthi M, Michel D, Knecht H, Perrelet R, Neto I, et al. Long-term changes in bone metabolism, bone mineral density, quantitative ultrasound parameters, and fracture incidence after spinal cord injury: a cross-sectional observational study in 100 paraplegic men. Osteoporos Int. Mar 2004;15(3):180-9. Epub 2004/01/15. 
Authors' roles:

Study design and conduct: AGZ, MPG, AN and ES. Data collection: AGZ, MPG, MP, XFW, SI.

Responsibility for StrAx analysis: AGZ. Responsibility for FEA analysis: SKB Statistical analyses: XW, and AGZ. Drafting manuscript: AGZ, MPG, MRF and ES. Data interpretation and approving final version of manuscript: AGZ, MPA, AN, MP, XFW, SI, AKB, MRF and ES. 
Figure 1.Images of trabecular and cortical bone at the distal tibia Axial and axial-coronal views of distal tibia of a 28 years male with spinal cord injury (panels 1,2) and a healthy control (panels 3,4). Cortices are thin and porous. Trabeculae are thinned, disconnected with loss of homogeneity in their distribution.

Figure 2. Distal tibial total, cortical and trabecular volumetric bone mineral density (vBMD) and cortical porosity as a function of time since injury.

The regression lines were derived by plotting vBMD and cortical porosity from 0 to 50 months and then from 50 to 250 months. vBMD was lower and cortical porosity was higher at later than earlier follow up times.

Figure 3. Comparison of deficits total, cortical and trabecular volumetric bone mineral density (vBMD) at the distal tibia (black bar), distal fibula (deep grey bars) and distal radius (light grey bars) in men with tetraplegia and paraplegia. Deficits are expressed as standard deviations from the mean in age-matched controls.

Figure 4. Differences in loading-induced microstrain within the range usually encountered by a bone do not modify modelling or remodelling (green). When loading exceeds a strain threshold threatening fracture (red), modelling increases bone formation and bone volume restoring strains. When loading is reduced, as occurs in spinal cord injury, strains decrease below a threshold inhibiting remodeling (blue) result in initiation of unbalanced remodelling causing bone loss and a decrease in bone volume which restores strains. 
Table 1. Distal tibial microstructure in individuals with tetraplegia and paraplegia and controls.

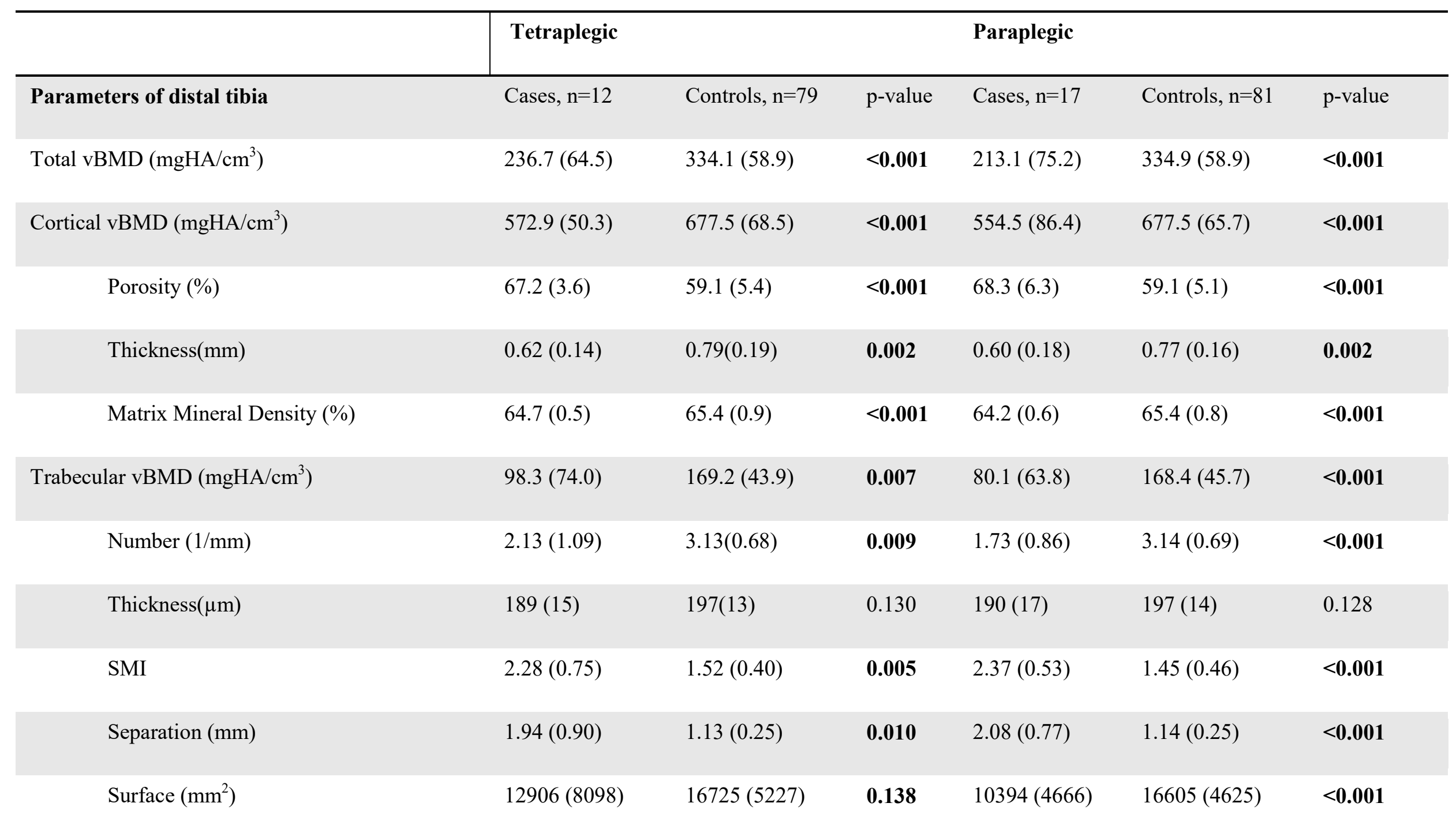




\begin{tabular}{|c|c|c|c|c|c|c|}
\hline Connectivity Density $\left(1 / \mathrm{mm}^{3}\right)$ & $2.83(2.11)$ & $4.56(1.54)$ & 0.017 & $2.14(1.43)$ & $4.38(1.49)$ & $<0.001$ \\
\hline Inhomogeneity (mm) & $0.51(0.48)$ & $0.21(0.08)$ & 0.053 & $0.72(0.61)$ & $0.21(0.08)$ & 0.003 \\
\hline
\end{tabular}


Table 2. Distal fibula microstructure in individuals with quadriplegia and paraplegia and controls

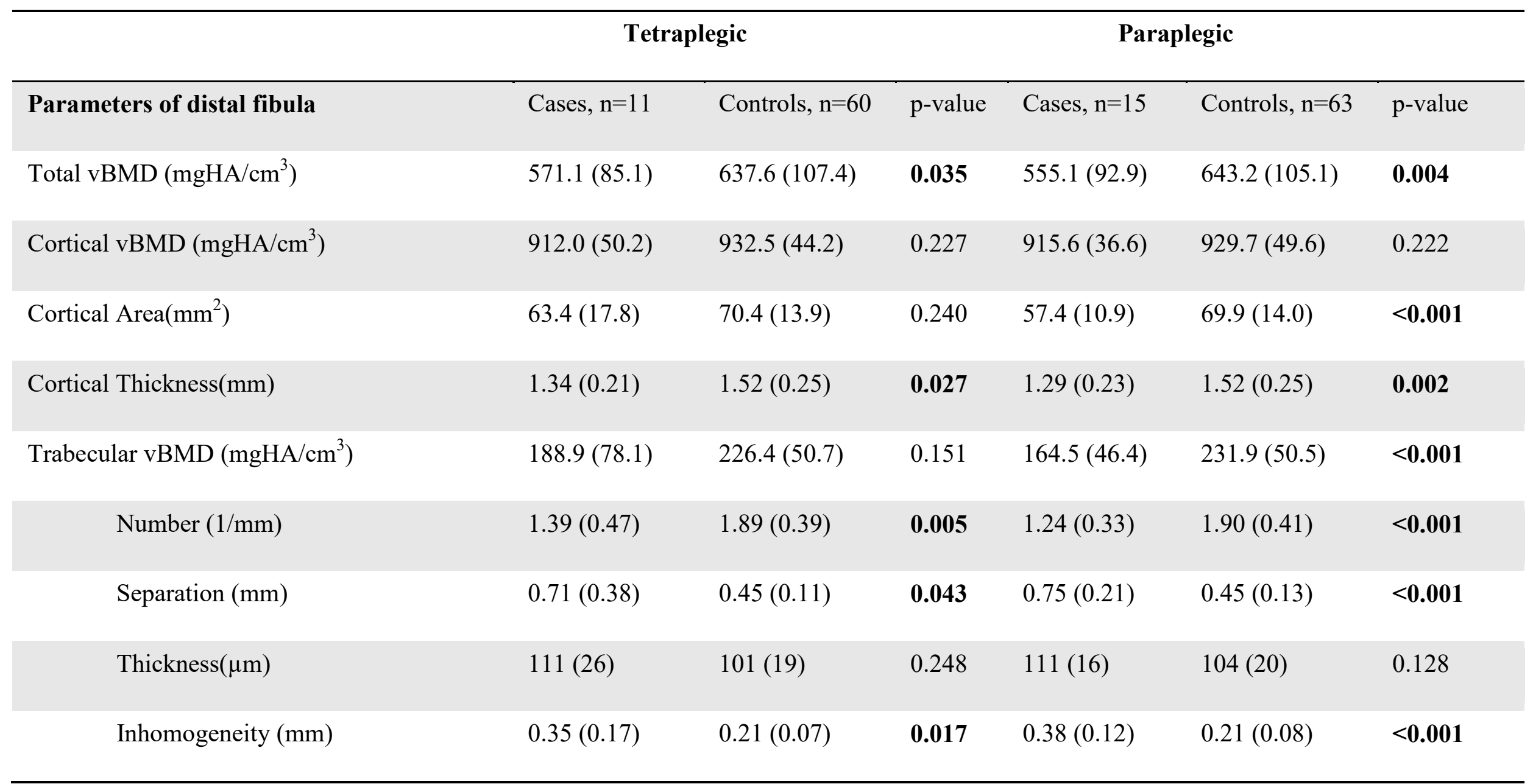

vBMD; Volumetric bone mineral density 
Table 3. Distal radial microstructure of individuals with tetraplegia and paraplegia and controls

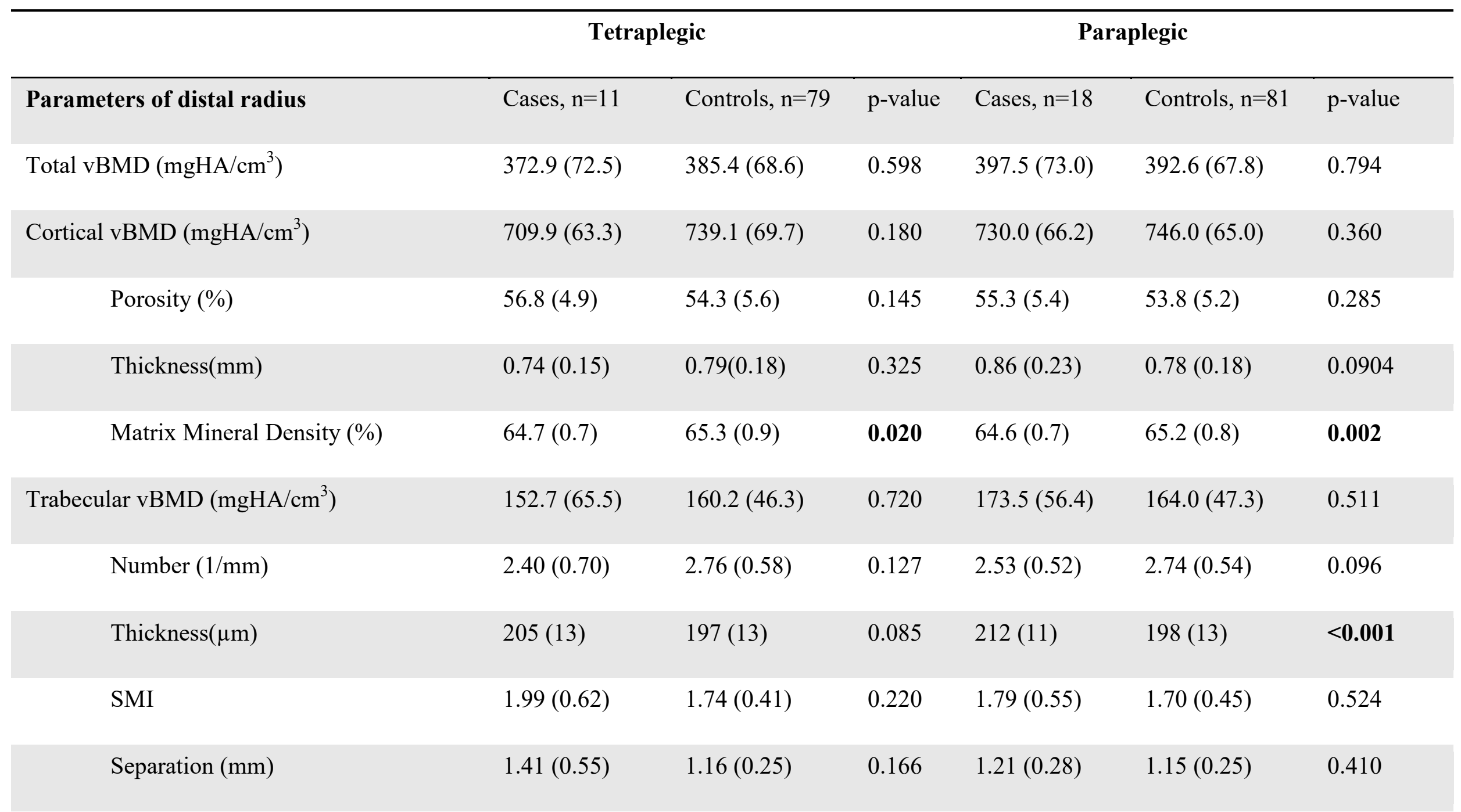




\begin{tabular}{|c|c|c|c|c|c|c|}
\hline Surface $\left(\mathrm{mm}^{2}\right)$ & 6408 (2489) & $6818(1786)$ & 0.608 & $6044(1242)$ & $6780(1783)$ & 0.044 \\
\hline Connectivity Density $\left(1 / \mathrm{mm}^{3}\right)$ & $3.61(1.37)$ & $4.46(1.21)$ & 0.073 & $4.01(0.95)$ & $4.35(1.18)$ & 0.200 \\
\hline Inhomogeneity (mm) & $0.25(0.14)$ & $0.19(0.07)$ & 0.190 & $0.21(0.07)$ & $0.19(0.06)$ & 0.272 \\
\hline Failure Load (N) & $2499(776)$ & $2668(652)$ & 0.526 & $2600(625)$ & $2680(662)$ & 0.651 \\
\hline
\end{tabular}

vBMD; Volumetric bone mineral density, SMI; Structure Model Index. SMI of 0 indicates plate-like structure, 3 indicates rod-like structure 
Table 4. Total, cortical and trabecular vBMD differences between tibia, fibula and radius in tetraplegic and paraplegic men.

Difference expressed as age-adjusted standardized deviations (SD) with $95 \%$ confidence intervals (CIs).

\begin{tabular}{|c|c|c|c|c|}
\hline Deviation & Tetraplegic & & Paraplegic & \\
\hline Total vBMD & Mean difference, SD (CI) & p-value & Mean difference, SD (CI) & p-value \\
\hline Tibia $v s$. Radius & $-1.51(-2.57,-0.45)$ & 0.007 & $-2.37(-3.22,1.52)$ & $<0.001$ \\
\hline \multicolumn{5}{|l|}{ Cortical vBMD } \\
\hline Tibia vs. Fibula & $-0.50(-1.10,0.10)$ & 0.096 & $-1.05(-1.69,-0.41)$ & 0.002 \\
\hline \multicolumn{5}{|l|}{ Trabecular vBMD } \\
\hline Tibia vs. Fibula & $-0.98(-2.51,0.55)$ & 0.198 & $-1.03(-2.07,0.01)$ & 0.051 \\
\hline Tibia vs. Radius & $-1.73(-3.25,-0.21)$ & 0.027 & $-2.66(-3.64,-1.68)$ & $<0.001$ \\
\hline
\end{tabular}




\section{Highlights}

- Weight-bearing regions have more severely compromised bone density and microstructure than less- or non-weight bearing regions after spinal cord injury.

- Higher cortical porosity, fewer and more separated trabeculae, with loss of trabecular homogeneity and lower matrix mineral density occur at weight-bearing regions following spinal cord injury.

- Uncompromised distal radial microstructure occurs in men despite tetraplegia suggesting this non-weight bearing region is adapted to low customary strains. 

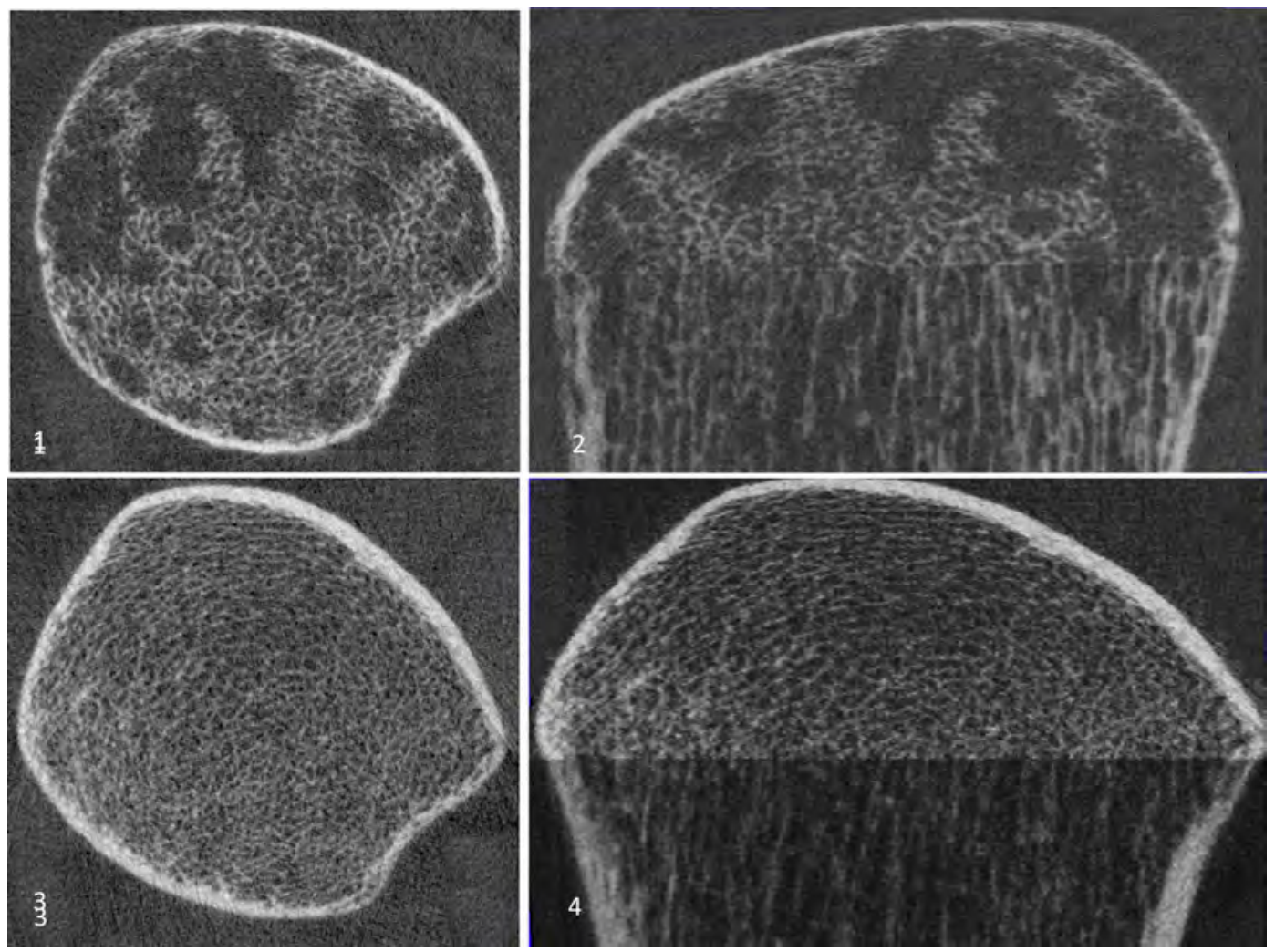

Figure 1 

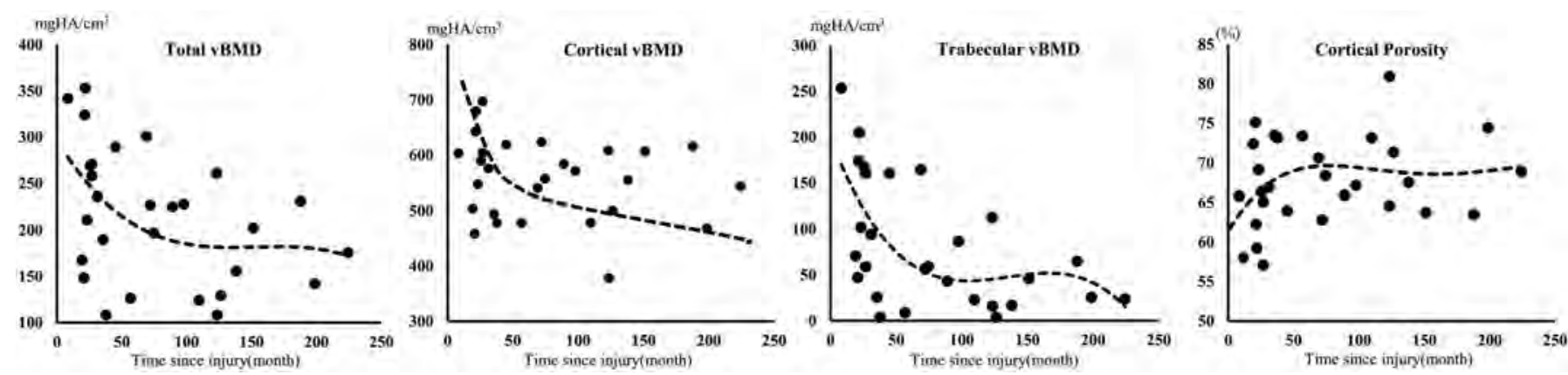

Figure 2 


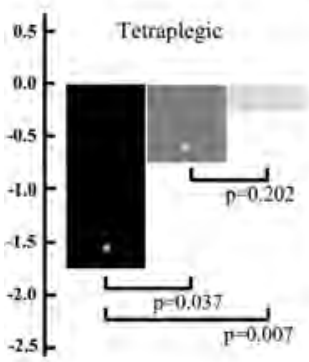

Paraplegic

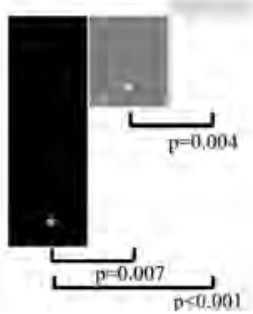

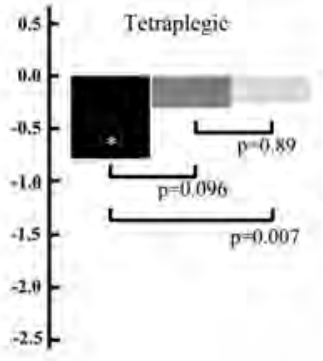

Paraplegic

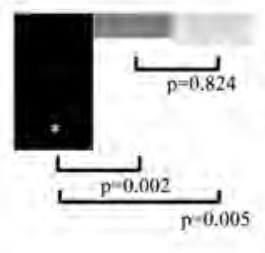

Paraplegic

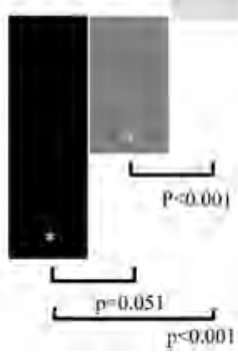

Figure 3 


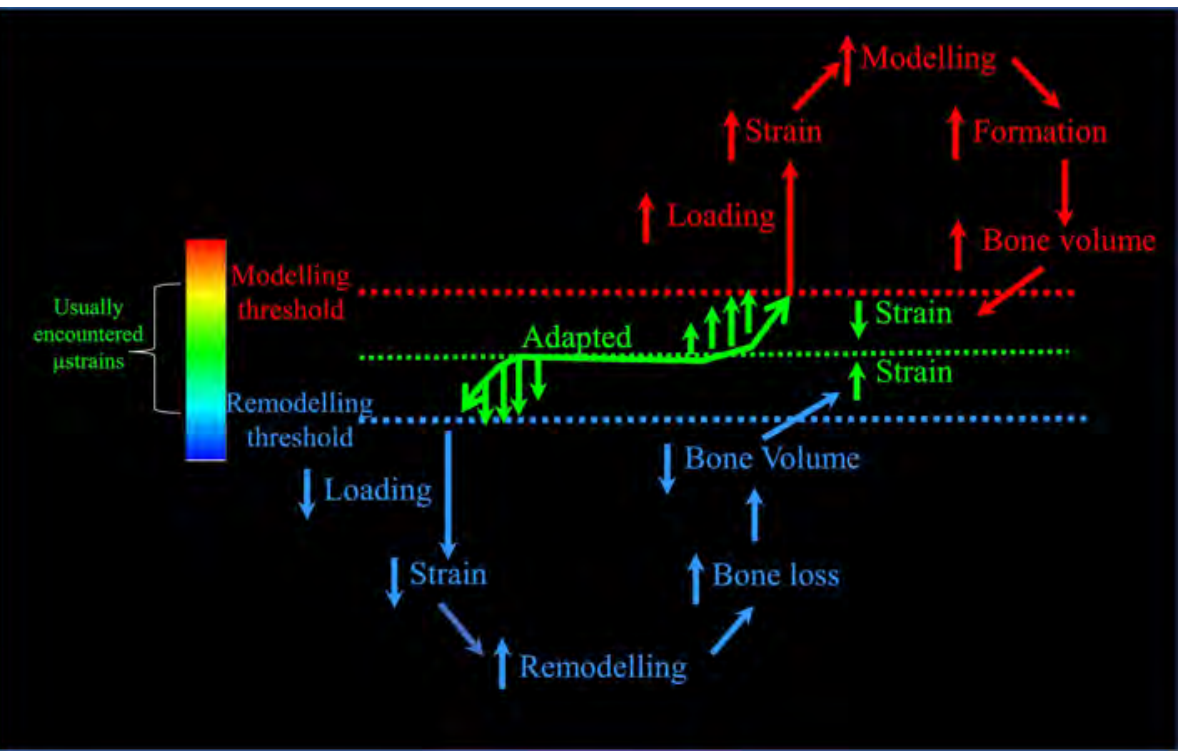

Figure 4 